Pacific Journal of Mathematics

AN INVERSION FORMULA FOR A DISTRIBUTIONAL 


\title{
AN INVERSION FORMULA FOR A DISTRIBUTIONAL FINITE-HANKEL-LAPLACE TRANSFORMATION
}

\author{
B. R. Bhonsle and R. A. Prabhu
}

In this paper a Finite-Hankel-Laplace transformation of a certain generalized functions is defined, and an inversion formula is established.

1. Introduction. Schwartz first introduced the Fourier transform of distributions in 1947. Since then, extension of the classical integral transformation to generalized functions has been of continuing interest. Some pertinent references are [1], [2], [3], [4], [5], [7], [8], and [9]. The classical Finite-Hankel-Laplace transform of function $f$ defined on $-\infty<x<\infty, 0<y<1$ is defined as

$$
F\left(s, j_{m}\right)=\int_{0}^{1} \int_{-\infty}^{\infty} y e^{-s x} J_{n}\left(j_{m} y\right) f(x, y) d x d y
$$

where $J_{n}(z)$ is the Bessel function of first kind of order $n \geqq-1 / 2$ and $j_{1}, j_{2}, j_{3} \cdots$ are positive zeros of $J_{n}(z)$ arranged in ascending order.

An inversion theorem for the transform (1.1) is as follows.

Theorem 1.1. Let $f(x, y)$ satisfy Dirichlet conditions in the interval $-\infty<x<\infty, 0<y<1$ and $y e^{-c x} f(x, y)$ be absolutely integrable on $-\infty<x<\infty, 0<y<1$ for some positive value of $c$. If its Finite-Hankel-Laplace transform in that range is defined as (1.1), then at any point $(x, y),-\infty<x<\infty, 0<y<1$ at which the function $f(x, y)$ is continuous,

$$
f(x, y)=\frac{1}{2 \pi i} \sum_{m=1}^{\infty} \frac{2 J_{n}\left(j_{m} y\right)}{J_{n+1}^{2}\left(j_{m}\right)} \int_{\sigma-i \infty}^{\sigma+i \infty} e^{s x} F\left(s, j_{m}\right) d s
$$

where $\operatorname{Re}(s)=\sigma>c$.

It is natural to try to extend the classical Finite-Hankel-Laplace transform to generalized functions. In this paper above Theorem 1.1 is extended to generalized functions.

2. The notation and terminology. In notation and terminology we follow [2] and [10]. The open set $(-\infty, \infty) \times(0,1)$ will be denoted by $I$. We will use the following operators,

$$
D_{x}^{k} \Omega_{n, y}^{k^{\prime}}=D_{x}^{k}\left(D_{y}^{2}+\frac{1}{y} D y-\frac{n^{2}}{y^{2}}\right)^{k^{\prime}}, k, k^{\prime}=0,1,2,3, \cdots
$$


where $n \geqq-1 / 2$ and the expression,

$$
T_{N}(y, \tau)=\sum_{m=1}^{N} \frac{2 J_{n}\left(j_{m} y\right) J_{n}\left(j_{m} \tau\right)}{J_{n+1}^{2}\left(j_{m}\right)}
$$

3. The spaces $L U_{a, b, c, n}, L U(w, z, c, n)$ and their duals. Let $a, b, c, n$ be real numbers such that $c \geqq 1 / 2, n \geqq-1 / 2$, and let $k_{a, b}^{(x)}$ be the function defined as

$$
k_{a, b}^{(x)}= \begin{cases}e^{a x} & 0 \leqq x<\infty \\ e^{b x} & -\infty<x<0\end{cases}
$$

then $L U_{a, b, c, n}$ is defined as the linear space of all complex valued smooth functions $\phi(x, y)$ on $-\infty<x<\infty, 0<y<1$ such that for each $k, k^{\prime}=0,1,2,3, \cdots$

$$
\rho_{a, b, k, k^{\prime}}^{c, n}[\phi(x, y)]=\sup _{\substack{-\infty<x<\infty \\ 0<y<1}}\left|k_{a, b}^{(x)} y^{c} D_{x}^{k} \Omega_{n, y}^{k^{\prime}}\left[y^{-1} \phi(x, y)\right]\right|<\infty .
$$

We assign to $L U_{a, b, c, n}$ the topology generated by the semi-norms $\left\{\rho_{a, b, k, k^{\prime}}^{c, n}\right\}_{k, k^{\prime}=0}$. Hence $L U_{a, b, c, n}$ is countably multinormed space which is complete. The dual space $L U_{a, b, c, n}^{\prime}$ consists of all continuous linear functionals on $L U_{a, b, c, n}$. By the Theorem 1.8.3 [10, p. 21] $L U_{a, b, c, n}^{\prime}$ is also complete. If $a \leqq d$ and $e \leqq b$, then $L U_{d, e, c, n} \subset L U_{a, b, c, n}$, and the topology of $L U_{d, e, c, n}$ is stronger than the topology induced on it by $L U_{a, b, c, n}$. Consequently the restriction of any member $f \in L U_{a, b, c, n}^{\prime}$ to $L U_{d, e, c, n}$ is in $L U_{d, e, c, n}^{\prime}$.

We turn now to a certain countable union space $L U(w, z, c, n)$. Let $w$ denote either a finite real number or $-\infty$ and $z$ denote either a finite real number or $+\infty$. Choose two monotonic sequences $\left\{a_{k}\right\}_{k=1}^{\infty}$ and $\left\{b_{k}\right\}_{k=1}^{\infty}$ such that $a_{k} \rightarrow w_{+}$and $b_{k} \rightarrow z_{-}$. Then $L U(w, z, c, n)$ is defined as countable union space of all $L U_{a_{k}, b_{k}, c, n}$ spaces; thus $L U(w, z, c, n)=\bigcup_{k=1}^{\infty} L U_{a_{k}, b_{k}, c, n}$. A sequence $\left\{\phi_{k}\right\}_{k=1}^{\infty}$ converges in $L U(w, z, c, n)$ if and only if it converges in $L U_{a_{k}, b_{k}, c, n}$ for some $k$. Since for each $k, L U_{a_{k}, b_{k}, c, n}$ is complete and hence a countable-union space, $L U(w, z, c, n)$ is complete. $L U^{\prime}(w, z, c, n)$ denotes the dual space of $L U(w, z, c, n)$. Hence $L U^{\prime}(w, z, c, n)$ is also complete [10, p. 25].

Now we note several facts to which we will refer later.

( I ) Clearly, $D(I)$ is sub-space of $L U_{a, b, c, n}$ as well as of $L U(w, z$, $c, n)$, whatever be the value of $a, b, w$ or $z$; the convergence in $D(I)$ implies the convergence in $L U_{a, b, c, n}$ and also convergence in $L U(w, z$, $c, n)$. Consequently, the restriction of any member of $L U_{a, b, c, n}^{\prime}$ or $L U^{\prime}(w, z, c, n)$ to $D(I)$ is a member of $D^{\prime}(I)$. Hence the member of $L U_{a, b, c, n}^{\prime}$ and $L U^{\prime}(w, z, c, n)$ are distributions in the sense of Zemanian $[10$, p. 39]. 
(II) Since $D(I)$ is dense in $L U(w, z, c, n)$ for every $w, z$ therefore by Theorem 1.9.1 [10, p. 24] $L U^{\prime}(w, z, c, n)$ is a subspace of $D^{\prime}(I)$.

(III) Let $w \leqq x$ and $y \leqq z$, then $L U(x, y, c, n) \subset L U(w, z, c, n)$ and convergence in $L U(x, y, c, n)$ implies the convergence in $L U(w, z, c, n)$. Since $D(I) \subset L U(x, y, c, n)$ and $D(I)$ is dense in $L U(w, z, c, n), L U(x$, $y, c, n)$ is also dense in $L U(w, z, c, n)$. Hence by Theorem 1.9.1 [10, p. 24] $L U^{\prime}(w, z, c, n)$ is a subspace of $L U^{\prime}(x, y, c, n)$.

(IV) If $f(x, y)$ is locally integrable function defined on $-\infty<$ $x<\infty, 0<y<1$ and if $\int_{0}^{1} \int_{-\infty}^{\infty}\left|\left[k_{a, b}^{(x)}\right]^{-1} y^{1-c} f(x, y)\right| d x d y$ exists, then $f(x, y)$ generates a regular generalized function on $L u_{a, b, c, n}^{\prime}$ through the definition

$$
\langle f, \phi\rangle=\int_{0}^{1} \int_{-\infty}^{\infty} f(x, y) \phi(x, y) d x d y, \phi \in L U_{a, b, c, n} .
$$

Similarly, if $w<a$ and $b<z$, then $f$ generates a regular member of $L U^{\prime}(w, z, c, n)$ through the definition

$$
\langle f, \phi\rangle=\int_{0}^{1} \int_{-\infty}^{\infty} f(x, y) \phi(x, y) d x d y, \phi \in L U(w, z, c, n) .
$$

(V) For each $m=1,2,3, \cdots$ and $n \geqq-1 / 2, c \geqq 1 / 2$ and $a \leqq$ $\operatorname{Re}(s) \leqq b$, the function $e^{-s x} y J_{n}\left(j_{m} y\right)$ is a member of $L U_{a, b, c, n}$.

For all $a>w$ and $z>b, e^{-s x} y J_{n}\left(j_{m} y\right)$ is a member of $L U(w, z, c, n)$.

4. The generalized Finite-Hankel-Laplace transformation. Let $c, n$ satisfy $n \geqq-1 / 2, c \geqq 1 / 2$. We shall call a generalized function $f$ as Finite-Hankel-Laplace-transformable if it belongs to $L U^{\prime}(w, z, c, n)$ for some real number $w, z$. Let $\sigma_{f}$ and $\rho_{f}$ defined as follows:

$$
\begin{aligned}
& \sigma_{f}=\inf \left\{w / f \in L U^{\prime}(w, z, c, n)\right\} \\
& \rho_{f}=\sup \left\{z / f \in L U^{\prime}(w, z, c, n)\right\} .
\end{aligned}
$$

We are now in a position to define the generalized Finite-HankelLaplace transformation, which we denoted by $\mathscr{L} \mathscr{H}_{n}$. For given Finite-Laplace-transformable generalized function $f$, let $\Omega_{f}$ denote the strip $\left\{s / \sigma_{f}<\operatorname{Re}(s)<\rho_{f}\right\}$ and let $\left\{j_{m}\right\}$ be the positive zeros of $J_{n}(z)$ arranged in ascending order. Then, the Finite-Hankel-Laplace transform $F\left(s, j_{m}\right)$ of $f$ is defined as the application of $f$ to the kernel $e^{-s x} y J_{n}\left(j_{m} y\right)$, i.e.,

$$
\mathscr{L} \mathscr{H}_{n}(f)\left(s, j_{m}\right)=F\left(s, j_{m}\right)=\left\langle f(x, y), e^{-s x} y J_{n}\left(j_{m} y\right)\right\rangle
$$

where $s \in \Omega_{f}$ and $\left\{j_{m}\right\}$ are the positive zeros of $J_{n}(z)$. For any $s \in \Omega_{f}$ and all $j_{m}$, the right-hand-side of (4.1) has meaning as the application of $f \in L U^{\prime}\left(\sigma_{f}, \rho_{f}, c, n\right)$ to $y e^{-s x} J_{n}\left(j_{m} y\right) \in L U\left(\sigma_{f}, \rho_{f}, c, n\right)$ (or equivalently 
the application of $f \in L U_{a, b, c, n}^{\prime}$ to $y e^{-s x} J_{n}\left(j_{m} y\right) \in L U_{a, b, c, n}$ for any $\sigma_{f}<$ $\left.a \leqq \operatorname{Re}(s) \leqq b<\rho_{f}\right)$.

If $f(x, y)$ is a locally integrable function such that $y e^{-s x} J_{n}\left(j_{m} y\right)$ is absolutely integrable and $\sigma_{f}<a<b<\rho_{f}$ then its conventional Finite-Hankel-Laplace transform

$$
\int_{0}^{1} \int_{-\infty}^{\infty} y e^{-s x} J_{n}\left(j_{m} y\right) f(x, y) d x d y
$$

exists for at least one $s \in \Omega_{f}$ and for all $j_{m^{\prime}}$ where $\left\{j_{m}\right\}$ are positive zeros of $J_{n}(z)$ and can be identified with our generalized Finite-HankelLaplace transform (4.1).

5. Inversion and uniqueness. We shall now derive an inversion formula for Finite-Hankel-Laplace-tranformation. The proof of the inversion formula requires some lemmas.

LeMma 5.1. Let $\mathscr{L} \mathscr{C}_{n}(f)=F\left(s, j_{m}\right)$ for $s \in \Omega_{f}$ and $j_{m}$, let $\phi(x, y) \in D(I)$, and set for $0<a^{\prime}<b^{\prime}<1$

$$
\Phi\left(s, j_{m}\right)=\int_{a^{\prime}}^{b^{\prime}} \int_{-\infty}^{\infty} y e^{+s x} J_{n}\left(j_{m} y\right) \phi(x, y) d x d y .
$$

Then for any fixed real number $R, 0<R<\infty$

$$
\begin{aligned}
& \int_{-R}^{R}\left\langle f(t, \tau), e^{-s t} \tau J_{n}\left(j_{m} \tau\right)\right\rangle \Phi\left(s, j_{m}\right) d w \\
& \quad=\left\langle f(t, \tau), \int_{-R}^{R} e^{-s t} \tau J_{n}\left(j_{m} \tau\right) \Phi\left(s, j_{m}\right) d w\right\rangle
\end{aligned}
$$

where $s=\sigma+i w$ and $\sigma$ is fixed with $\sigma_{f}<\sigma<\rho_{f}$.

Proof. As it is obvious that the functions $e^{-s t} \tau J_{n}\left(j_{m} \tau\right)$ and $\int_{-R}^{R} e^{-s t} \tau J_{n}\left(j_{m} \tau\right) \Phi\left(s, j_{m}\right) d w$ are members of the space $L U_{a, b, c, n}$ with $t, \tau$ as the variables of testing functions, the expressions on both the sides of (5.1) have sense. Using the technique of Riemann sums as used in [10, Lemma 3.5.1. p. 64], (5.1) can be easily established.

Lemma 5.2. Let $a^{\prime}, b^{\prime}$ be any two real numbers satisfying $0<$ $a^{\prime}<b^{\prime}<1$. Then

$$
\lim _{R, N \rightarrow \infty} \int_{a^{\prime}}^{b^{\prime}} \int_{-\infty}^{\infty} y T_{N}(y, \tau) \frac{\operatorname{Sin} R(x-t)}{(x-t)} d x d y=\pi
$$

when $-\infty<t<\infty, a^{\prime}<\tau<b^{\prime}$.

The proof can be left to the reader. 
LEMMA 5.3. Let $a, b, c$ and $R$ be real numbers such that $a<\sigma<b$ and $c \geqq 1 / 2$, let $\psi(x, y) \in D(I)$. Then for fixed $y, \psi(x, y) \in D\left(I_{y}\right) I_{y}=$ $\{(x, y) /-\infty<x<\infty, y$ is fixed $\}$

$$
\frac{1}{\pi} \int_{-\infty}^{\infty} \psi(x, y) e^{o(x-t)} \frac{\operatorname{Sin} R(x-t)}{(x-t)} d x
$$

converges in $L U_{a, b, c, n}$ to $\psi(t, y)$ as $R \rightarrow \infty$.

The proof is similar to that of Lemma 3.5.2 [10, p. 66].

Lemma 5.4. If $\psi(x, y) \in D(I)$, then

$$
\begin{aligned}
& \frac{1}{\pi} k_{a, b}^{(t)} \tau^{c} \int_{a^{\prime}}^{b^{\prime}} \int_{-\infty}^{\infty}\left[e^{\sigma(x-t)} \psi(x, y)\right. \\
& \quad-\psi(t, \tau)] y T_{N}(y, \tau) \frac{\operatorname{Sin} R(x-t)}{(x-t)} d x d y
\end{aligned}
$$

converges to zero uniformly as $R, N \rightarrow \infty$ for all $(t, \tau) \in(-\infty, \infty) \times$ $(0,1)$, where the support of $\psi(x, y)$ is contained in $[A, B] \times\left[a^{\prime}, b^{\prime}\right]$; where $-\infty<A<B<\infty, 0<a^{\prime}<b^{\prime}<1$.

Proof. Let us divide the interval $(-\infty, \infty) \times(0,1)$ into four disjoint sets $[(-\infty, A) U(B, \infty)] \times(0,1),(A, B) \times\left(b^{\prime}, 1\right),(A, B) \times\left(0, a^{\prime}\right)$ and $[A, B] \times\left[a^{\prime}, b^{\prime}\right]$. For $(t, \tau) \in[(-\infty, A) U(B, \infty)] \times(0,1), \psi(t, \tau)=0$ since $\psi(t, \tau)$ is supported by $[A, B] \times\left[a^{\prime}, b^{\prime}\right]$.

Therefore

$$
\begin{gathered}
\frac{1}{\pi} k_{a, b}^{(t)} \tau^{c} \int_{a^{\prime}}^{b^{\prime}} \int_{-\infty}^{\infty}\left[e^{\sigma(x-t)} \psi(x, y)-\psi(t, \tau)\right] y T_{N}(y, \tau) \frac{\operatorname{Sin} R(x-t)}{(x-t)} d x d y \\
=\frac{1}{\pi} k_{a, b}^{(t)} \tau^{c} \int_{a^{\prime}}^{b^{\prime}} \int_{-\infty}^{\infty} e^{\sigma(x-t)} \psi(x, y) y T_{N}(y, \tau) \frac{\operatorname{Sin} R(x-t)}{(x-t)} d x d y .
\end{gathered}
$$

In view of Lemma 5.3 as $R \rightarrow \infty$, this integral reduces to

$$
\frac{1}{\pi} k_{a, b}^{(t)} \tau^{c} \int_{a^{\prime}}^{b^{\prime}} y \psi(t, y) T_{N}(y, \tau) d y \text {. }
$$

Thus we want to show that for fixed $t$ and $0<y<1$

$$
\lim _{N \rightarrow \infty} \frac{1}{\pi} k_{a, b}^{(t)} \tau^{c} \int_{a^{\prime}}^{b^{\prime}} y \psi(t, y) T_{N}(y, \tau) d y=0
$$

uniformly for all $(t, \tau)$. Since $\psi(t, y) \in D\left(I_{t}\right)$,

$$
I_{t}=\{(t, y) / t \text { is fixed }, \quad 0<y<1\}
$$

then $\psi(t, y)$ is bounded say by $K$ 


$$
\begin{array}{r}
\left|k_{a, b}^{(t)} \tau^{c} \int_{a^{\prime}}^{b^{\prime}}\left[\psi(t, y) y T_{N}(y, \tau)\right] d y\right| \\
\leqq K \tau^{c} k_{a, b}^{(t)} \int_{a}^{b \prime}\left|y T_{N}(y, \tau)\right| d y
\end{array}
$$

In view of the analogue of Riemann Lebesque lemma [6, p. 589], for given $\varepsilon>0$ there exists a positive integer $N_{0}$ such that

$$
\left|\int_{a^{\prime}}^{b^{\prime}} y T_{N}(y, \tau) d y\right| \leqq \frac{8 c_{1}^{2} \varepsilon}{\pi c_{2}^{2}\left(2-\tau-b^{\prime}\right) \sqrt{\tau}}
$$

for all $N \geqq N_{0}$, which is bounded by $c^{\prime} \varepsilon /\left(1-b^{\prime}\right) \sqrt{\tau}$. Therefore, for all $N \geqq N_{0}$ and for all $(t, \tau) \in[(-\infty, A) U(B, \infty)] \times(0,1)$

$$
k_{a, b}^{(t)} \tau^{c}\left|\int_{a^{\prime}}^{b^{\prime}} y \psi(t, y) T_{N}(y, \tau) d y\right| \leqq \frac{c^{\prime} \in \tau^{c-1 \mid 2}}{\left(1-b^{\prime}\right)} \leqq \frac{c^{\prime} \varepsilon}{\left(1-b^{\prime}\right)}
$$

since $c \geqq 1 / 2$. Hence as $\varepsilon$ is arbitrary, we have (5.5) as stated above.

Thus

$$
\begin{aligned}
& \frac{1}{\pi} k_{a, b}^{(t)} \tau^{c} \int_{a^{\prime}}^{b^{\prime}} \int_{-\infty}^{\infty}\left[e^{\sigma(x-t)} \psi(x, y)\right. \\
& \quad-\psi(t, \tau)] y T_{N}(y, \tau) \frac{\operatorname{Sin} R(x-t)}{(x-t)} d x d y \longrightarrow 0
\end{aligned}
$$

as $R, N \rightarrow \infty$, uniformly for all $(t, \tau) \in[(-\infty, A) U(B, \infty)] \times(0,1)$.

In a similar manner we can prove that for all $(t, \tau) \in(A, B) \times$ $\left(b^{\prime}, 1\right)$ and $(t, \tau) \in(A, B) \times\left(0, a^{\prime}\right)$

$$
\begin{aligned}
\frac{1}{\pi} k_{a, b}^{(t)} \tau^{c} \int_{a^{\prime}}^{b^{\prime}} \int_{-\infty}^{\infty}\left[e^{\sigma(x-t)} \psi(x, y)-\psi(t, \tau)\right] \\
\quad \times y T_{N}(y, \tau) \frac{\operatorname{Sin} R(x-t)}{(x-t)} d x d y \longrightarrow 0
\end{aligned}
$$

uniformly as $R, N \rightarrow \infty$.

Next we want to show that

$$
\begin{gathered}
\frac{1}{\pi} k_{a, b}^{(t)} \tau^{c} \int_{a^{\prime}}^{b^{\prime}} \int_{-\infty}^{\infty}\left[e^{\sigma(x-t)} \psi(x, y)-\psi(t, \tau)\right] \frac{\operatorname{Sin} R(x-t)}{(x-t)} \\
\times y T_{N}(y, \tau) d x d y \longrightarrow 0 \text { as } R, N \longrightarrow \infty
\end{gathered}
$$

uniformly for all $(t, \tau) \in[A, B] \times\left[a^{\prime}, b^{\prime}\right]$.

Now

$$
\begin{gathered}
\left.\frac{1}{\pi} k_{a, b}^{(t)} \tau^{c} \int_{a^{\prime}}^{b^{\prime}} \int_{-\infty}^{\infty} e^{\sigma(x-t)} \psi(x, y)-\psi(t, \tau)\right] \\
\times \frac{\operatorname{Sin} R(x-t)}{(x-t)} y T_{N}(y, \tau) d x d y
\end{gathered}
$$




$$
\longrightarrow \frac{1}{\pi} k_{a, b}^{(t)} \tau^{c} \int_{a^{\prime}}^{b^{\prime}}[\psi(t, y)-\psi(t, \tau)] y T_{N}(y, \tau) d y,
$$

$$
\text { as } R \longrightarrow \infty \text {. }
$$

Hence we need to show that for fixed $t$ and $a^{\prime}<\tau<b^{\prime}$

$$
\frac{1}{\pi} k_{a, b}^{(t)} \tau^{c} \int_{a^{\prime}}^{b^{\prime}}[\psi(t, y)-\psi(t, \tau)] y T_{N}(y, \tau) d y \longrightarrow 0 \quad \text { as } \quad N \longrightarrow \infty
$$

uniformly for all $(t, \tau)$. Let $F(y, t, \tau)\left(y^{2}-\tau^{2}\right)=y^{-n}[\psi(t, y)-\psi(t, \tau)]$ for $0<y<1,0<\tau<1$, and $t$ is fixed. Now define function

$$
\begin{aligned}
G(y, t, \tau) & =F(y, t, \tau), y=\tau \\
& =\frac{y^{-n} D \psi(t, y)}{2 y}, y \neq \tau, D=\frac{\partial}{\partial y},
\end{aligned}
$$

$G(y, t, \tau)$ is continuous of $y, t$, and $\tau$ in the domain $\{t$ is fixed, $0<$ $y<1,0<\tau<1$. Now

$$
\begin{aligned}
\int_{a^{\prime}}^{b^{\prime}} y & {[\psi(t, y)-\psi(t, \tau)] T_{N}(y, \tau) d y } \\
& =\int_{a^{\prime}}^{b^{\prime}} y^{n+1} F(y, t, \tau)\left(y^{2}-\tau^{2}\right) T_{N}(y, \tau) d y \\
& =\int_{a^{\prime}}^{b^{\prime}} y^{n+1} G(y, t, \tau)\left(y^{2}-\tau^{2}\right) T_{N}(y, \tau) d y
\end{aligned}
$$

as the value of the integral remains unchanged by replacing expression $f(y, t, \tau)\left(y^{2}-\tau^{2}\right)$ by $G(y, t, \tau)\left(y^{2}-\tau^{2}\right)$.

Let us now divide the interval $a^{\prime} \leqq \tau \leqq b^{\prime}$ in to $p$ equal parts by the points $a^{\prime}=y_{0}, y_{1}, \cdots, y_{p}=b^{\prime}$ and after choosing positive number $\varepsilon, p$ so large that

$$
\sum_{m=1}^{p}\left(U_{m}-L_{m}\right)\left(y_{m}-y_{m-1}\right)<\varepsilon
$$

where $U_{m}$ and $L_{m}$ are upper and lower bounds of $G(t, y, \tau)$ in $y_{m-1} \leqq$ $y \leqq y_{m}, a^{\prime} \leqq \tau \leqq b^{\prime}$. Let $G(t, y, \tau)=G\left(t, y_{m-1}, \tau\right)+w_{m}(t, y, \tau)$ for $y_{m-1} \leqq y \leqq y_{m}, a^{\prime} \leqq \tau \leqq b^{\prime}$ so that $\left|w_{m}(t, y, \tau)\right| \leqq U_{m}-L_{m}$.

Using uniform continuity of the function $G(t, y, \tau)$ over the region $\left\{a^{\prime} \leqq y \leqq b^{\prime}, a^{\prime} \leqq \tau \leqq b^{\prime}\right\}$ and following the lines in the proof of the analogue of Riemann Lebesgue lemma [6, p. 589], for an arbitrary $\varepsilon>0$ we get a positive integer $N_{1}$ such that

$$
\left|\int_{a^{\prime}}^{b^{\prime}} y^{n+1} G(t, y, \tau)\left(y^{2}-\tau^{2}\right) T_{N}(y, \tau) d y\right| \leqq \frac{c^{\prime} \cdot \varepsilon}{\left(1-b^{\prime}\right) \sqrt{\tau}}
$$

for all $N \geqq N_{1}$. Hence for all $(t, \tau), t$ is fixed and $a^{\prime}<\tau<b^{\prime}$ 


$$
\begin{aligned}
k_{a, b}^{(t)} \tau^{c} & \int_{a^{\prime}}^{b^{\prime}} y[\psi(t, y)-\psi(t, \tau)] T_{N}(y, \tau) d y \\
& \leqq \frac{c^{\prime} \in \tau^{c-1 \backslash 2}}{\left(1-b^{\prime}\right)} \leqq \frac{c^{\prime} \varepsilon}{\left(1-b^{\prime}\right)} \quad \text { where } \quad c \geqq \frac{1}{2}
\end{aligned}
$$

Since $\varepsilon$ is arbitrary,

$$
\frac{1}{\pi} k_{a, b}^{(t)} \tau^{c} \int_{a^{\prime}}^{b^{\prime}} y[\psi(t, y)-\varphi(t, \tau)] T_{N}(y, \tau) d y \longrightarrow 0 \quad \text { as } \quad N \longrightarrow \infty
$$

uniformly for all fixed $t$ and $\tau \in\left[a^{\prime}, b^{\prime}\right]$.

Thus

$$
\begin{array}{r}
\frac{1}{\pi} k_{a, b}^{(t)} \tau^{c} \int_{a^{\prime}}^{b^{\prime}} \int_{-\infty}^{\infty}\left[e^{\sigma(x-t)} \psi(x, y)-\psi(t, \tau)\right] \\
\frac{\operatorname{Sin} R(x-t)}{(x-t)} y T_{N}(y, \tau) d x d y \longrightarrow 0
\end{array}
$$

uniformly for all $(t, \tau) \in[A, B] \times\left[a^{\prime}, b^{\prime}\right]$ as $R, N \rightarrow \infty$. Combining (5.6), (5.7) and (5.8), the lemma yields.

Lemma 5.5. For $\phi(x, y) \in D(I), 0<a^{\prime}<b^{\prime}<1$

$$
\Phi\left(s, j_{m}\right)=\int_{a^{\prime}}^{b^{\prime}} \int_{-\infty}^{\infty} y e^{+s x} J_{n}\left(j_{m} y\right) \dot{\phi}(x, y) d x d y \text {. }
$$

$$
\tau M_{R, V}(t, \tau)=\tau\left[\sum_{m=1}^{N} \frac{2}{J_{n+1}^{2}\left(j_{m}\right)} \frac{1}{2 \pi} \int_{-R}^{R}\left[e^{-s t} J_{n}\left(j_{m} \tau\right) \Phi\left(s, j_{m}\right)\right] d w\right]
$$

converges in $L U_{a, b, c, n}$ to $\tau \phi(t, \tau)$ as $R, N \rightarrow \infty$ for all $(t, \tau) \in(-\infty, \infty) \times$ $(0,1)$.

Proof. Since the integrand in (5.10) is a smooth function and $\phi$ is of bounded support, we may differentiate under the integral sign, and obtain

$$
\begin{aligned}
D_{t}^{k} Q_{n, \tau}^{k^{\prime}}[ & {\left[\tau^{-1} \cdot \tau M_{R, N}(t, \tau)\right] } \\
= & \sum_{m=1}^{N} \frac{2}{J_{n+1}^{2}\left(j_{m}\right)}\left[\frac{1}{2 \pi} \int_{-R}^{R} D_{t}^{k} Q_{n, \tau}^{k^{\prime}}\left[e^{-s t} J_{n}\left(j_{m} \tau\right)\right] \Phi\left(s, j_{m}\right) d w\right] \\
= & \sum_{m=1}^{N} \frac{2}{J_{n+1}^{2}\left(j_{m}\right)}\left\{\frac{1}{2 \pi} \int_{-R}^{R}(-1)^{k+k^{\prime}} s^{k} j_{m}^{2 k^{\prime}} e^{-s t} J_{n}\left(j_{m} \tau\right)\right. \\
& \left.\times\left[\int_{a^{\prime}}^{b^{\prime}} \int_{-\infty}^{\infty} e^{s x} y J_{n}\left(j_{m} y\right) \phi(x, y) d x d y\right] d w\right\} \\
= & \sum_{m=1}^{N} \frac{2}{J_{n+1}^{2}\left(j_{m}\right)}\left\{\frac{1}{2 \pi} \int_{-R}^{R}(-1)^{k} e^{-s t} J_{n}\left(j_{m} \tau\right)\right. \\
& \left.\times\left[\int_{a^{\prime}}^{b^{\prime}} \int_{-\infty}^{\infty}(-1)^{k^{\prime}} s^{k} j_{m}^{2 k^{\prime}} y e^{s x} J_{n}\left(j_{m} y\right) \dot{\phi}(x, y) d x d y\right] d w\right\}
\end{aligned}
$$




$$
\begin{aligned}
= & \sum_{m=1}^{N} \frac{2}{J_{n+1}^{2}\left(j_{m}\right)}\left\{\frac{1}{2 \pi} \int_{-R}^{R}(-1)^{k} e^{-s t} J_{n}\left(j_{m} \tau\right)\right. \\
& \times\left[\left[_{a}^{b^{\prime}} \int_{-\infty}^{\infty} D_{x}^{k} Q_{n, y}^{k^{\prime}}\left[e^{s x} J_{n}\left(j_{m} y\right)\right] y \phi(x, y)^{-} d x d y\right] d w\right\} .
\end{aligned}
$$

Now we consider

$$
(-1)^{k} \int_{a^{\prime}}^{b^{\prime}} \int_{-\infty}^{\infty} D_{x}^{k} Q_{n, y}^{k^{\prime}}\left[e^{s x} J_{n}\left(j_{m} y\right)\right] y \phi(x, y) d x d y .
$$

Upon integrating by parts the inner integral $k$ times and since $\phi(x, y)$ is of compact support this integral reduces to

$$
\int_{a^{\prime}}^{b^{\prime}} y \Omega_{n, y}^{k^{\prime}} J_{n}\left(j_{m} y\right)\left[\int_{-\infty}^{\infty} D_{x}^{k}[\phi(x, y)] e^{s x} d x\right] d y .
$$

Again integrating by parts $2 k^{\prime}$ times we get

$$
\int_{a^{\prime}}^{b^{\prime}} \int_{-\infty}^{\infty} D_{x}^{k} Q_{n, y}^{k^{\prime}}[\phi(x, y)] y e^{s x} J_{n}\left(j_{m} y\right) d x d y
$$

Therefore

$$
\begin{gathered}
D_{t}^{k} \Omega_{n, \tau}^{k^{\prime}}\left[\tau^{-1} \cdot \tau M_{R, N}(t, \tau)\right]=\frac{1}{2 \pi} \sum_{m=1}^{N} \frac{2}{J_{n+1}^{2}\left(j_{m}\right)}\left\{\int_{-R}^{R} e^{-s t} J_{n}\left(j_{m} \tau\right)\right. \\
\left.\times\left[\int_{a^{\prime}}^{b^{\prime}} \int_{-\infty}^{\infty} D_{x}^{k} \Omega_{n, y}^{k^{\prime}}[\phi(x, y)] y e^{s x} J_{n}\left(j_{m} y\right) d x d y\right] d w\right\} .
\end{gathered}
$$

Changing the order of integration, we obtain

$$
\begin{aligned}
D_{t}^{k} Q_{n, \tau}^{k^{\prime}}\left[\tau^{-1} \tau M_{R, N}(t, \tau)\right] & \\
& =\frac{1}{2 \pi} \int_{a^{\prime}}^{b^{\prime}} \int_{-\infty}^{\infty}\left[D_{x}^{k} Q_{n, y}^{k^{\prime}}[\phi(x, y)] y T_{N}(y, \tau)\left[\int_{-R}^{R} e^{s(x-t)} d w\right] d x d y\right] \\
& =\frac{2}{2 \pi} \int_{a^{\prime}}^{b^{\prime}} \int_{-\infty}^{\infty} D_{x}^{k} \Omega_{n}^{k^{\prime}}{ }_{y}[\phi(x, y)] y T_{N}(y, \tau) \frac{\operatorname{Sin} R(x-t)}{(x-t)} e^{\sigma(x-t)} d x d y .
\end{aligned}
$$

Hence in light of Lemma 5.2 we have as $R, N \rightarrow \infty$

$$
\begin{aligned}
D_{t}^{k} \Omega_{n, \tau}^{k^{\prime}} \tau^{-1}\left[\tau M_{R, N}(t, \tau)-\tau \phi(t, \tau)\right] \\
=\frac{1}{\pi} \int_{a^{\prime}}^{b^{\prime}} \int_{-\infty}^{\infty}\left[e^{\sigma(x-t)} D_{x}^{k} Q_{n, y}^{k^{\prime}}[\phi(x, y)]-D_{t}^{k} Q_{n, \tau}^{k^{\prime}}[\phi(t, \tau)]\right] \\
\quad \times \frac{\operatorname{Sin} R(x-t)}{(x-t)} y T_{N}(y, \tau) d x d y \\
=\frac{1}{\pi} \int_{a^{\prime}}^{b^{\prime}} \int_{-\infty}^{\infty}\left[e^{\sigma(x-t)} \psi(x, y)-\psi(t, \tau)\right] \frac{\operatorname{Sin} R(x-t)}{(x-t)} y T_{N}(y, \tau) d x d y
\end{aligned}
$$

where $\psi(x, y)=D_{x}^{k} \Omega_{n, y}^{k^{\prime}}[\phi(x, y)]$ which is again a member of $D(I)$ with support contained in $[A, B] \times\left[a^{\prime}, b^{\prime}\right]$. Hence it suffices to show that 


$$
\begin{gathered}
\frac{1}{\pi} k_{a, b}^{(t)} \tau^{c}\left[\int_{a^{\prime}}^{b^{\prime}} \int_{-\infty}^{\infty}\left[e^{\sigma(x-t)} \psi(x, y)-\psi(t, \tau)\right]\right] \\
\times \frac{\operatorname{Sin} R(x-t)}{(x-t)} y T_{N}(y, \tau) d x d y
\end{gathered}
$$

converges to zero as $R, N \rightarrow \infty$ uniformly for all $(t, \tau) \in(-\infty, \infty) \times$ $(0,1)$. This is true in view of Lemma 5.4, and thus the proof of Lemma 5.5 is complete.

6. The main theorem. Let $f(x, y)$ be a Finite-Hankel-Laplacetransformable function and $F\left(s, j_{m}\right)$ the generalized Finite-HankelLaplace-transform of $f$ as defined by

$$
F\left(s, j_{m}\right)=\left\langle f(x, y), e^{-s x} y J_{n}\left(j_{m} y\right)\right\rangle
$$

for $s \in \Omega_{f}$ and $\left\{j_{m}\right\}$, the positive zeros of $J_{n}(z)$.

Then in the sense of convergence in $D^{\prime}(I)$

$$
f(x, y)=\lim _{R, N \rightarrow \infty} \frac{1}{2 \pi i} \sum_{m=1}^{N} \frac{2 J_{n}\left(j_{m} y\right)}{J_{n+1}^{2}\left(j_{m}\right)} \int_{\sigma-i R}^{\sigma+i R} e^{s x} F\left(s, j_{m}\right) d s
$$

where $\sigma$ is any fixed number $\sigma_{f}<\sigma<\rho_{f}$.

Proof. Let $\phi(x, y)$ be an arbitrary member of $D(I)$. We wish to show that

$$
\begin{array}{r}
\left\langle\frac{1}{2 \pi i} \sum_{m=1}^{N} \frac{2 J_{n}\left(j_{m} y\right)}{J_{n+1}^{2}\left(j_{m}\right)} \int_{\sigma-i R}^{\sigma+i R} e^{s x} F\left(s, j_{m}\right) d s, \phi(x, y)\right\rangle \\
=\langle f(t, \tau), \phi(t, \tau)\rangle \text { as } R, N \longrightarrow \infty .
\end{array}
$$

Since $\phi(x, y) \in D(I)$ iff $y \phi(x, y) \in D(I)$, then (6.2) will be equivalent to showing that

$$
\begin{gathered}
\left\langle\frac{1}{2 \pi i} \sum_{m=1}^{N} \frac{2 J_{n}\left(j_{m} y\right)}{J_{n+1}^{2}\left(j_{m}\right)} \int_{\sigma-i R}^{\sigma+i R} e^{s x} F\left(s, j_{m}\right) d s, y \dot{\phi}(x, y)\right\rangle \\
=\langle f(t, \tau), \tau \phi(t, \tau)\rangle \text { as } \quad R, N \longrightarrow \infty .
\end{gathered}
$$

As $\phi(x, y) \in D(I)$, let us assume that the support of $\phi(x, y)$ is contained in $[A, B] \times\left[a^{\prime}, b^{\prime}\right]$, where $-\infty<A<B<\infty, 0<a^{\prime}<b^{\prime}<1$. As

$$
\frac{1}{2 \pi i} \sum_{m=1}^{N} \frac{2 J_{n}\left(j_{m} y\right)}{J_{n+1}^{2}\left(j_{m}\right)} \int_{\sigma-i R}^{o+i R} e^{s x} F\left(s, j_{m}\right) d s
$$

is locally integrable and since $y \dot{\phi}(x, y) \in D(I)$ then without limit notation (6.3) can be written as

$$
\int_{a^{\prime}}^{b^{\prime}} \int_{-\infty}^{\infty} y \phi(x, y)\left[\sum_{m=1}^{N} \frac{2 J_{n}\left(j_{m} y\right)}{J_{n+1}^{2}\left(j_{m}\right)} \frac{1}{2 \pi i} \int_{\sigma-i R}^{\sigma+i R} e^{s x} F\left(s, j_{m}\right) d s\right] d x d y
$$


Put $s=\sigma+i w$. We get

$$
\int_{a^{\prime}}^{b^{\prime}} \int_{-\infty}^{\infty} y \phi(x, y)\left[\sum_{m=1}^{N} \frac{2 J_{n}\left(j_{m} y\right)}{J_{n+1}^{2}\left(j_{m}\right)} \frac{1}{2 \pi} \int_{-R}^{R} e^{s x} F\left(s, j_{m}\right) d w\right] d x d y
$$

Since $\phi(x, y)$ has a compact support and the integrand is a continuous function of $(x, y, w)$ we can interchange the order of integration.

$$
\begin{aligned}
\sum_{m=1}^{N} \frac{2}{J_{n+1}^{2}\left(j_{m}\right)}\left\{\frac{1}{2 \pi} \int_{-R}^{R}\left\langle f(t, \tau), e^{-s t} \tau J_{n}\left(j_{m} \tau\right)\right\rangle\right. \\
\left.\quad \times\left[\int_{a^{\prime}}^{b^{\prime}} \int_{-\infty}^{\infty} e^{s x} J_{n}\left(j_{m} y\right) y \phi(x, y) d x d y\right] d w\right\} \\
=\sum_{m=1}^{N} \frac{2}{J_{n+1}^{2}\left(j_{m}\right)}\left\{\frac{1}{2 \pi} \int_{-R}^{R}\left\langle f(t, \tau), e^{-s t} \tau J_{n}\left(j_{m} \tau\right)\right\rangle \Phi\left(s, j_{m}\right) d w\right\}
\end{aligned}
$$

where

$$
\Phi\left(s, j_{m}\right)=\int_{a^{\prime}}^{b^{\prime}} \int_{\infty}^{\infty} e^{s x} y J_{n}\left(j_{m} y\right) \phi(x, y) d x d y
$$

Now by Lemma 5.1 we have

$$
\begin{aligned}
& \sum_{m=1}^{N} \frac{2}{J_{n+1}^{2}\left(j_{m}\right)}\left\{\frac{1}{2 \pi} \int_{-R}^{R}\left[\left\langle f(t, \tau), e^{-s t} \tau J_{n}\left(j_{m} \tau\right)\right\rangle \Phi\left(s, j_{m}\right)\right] d w\right\} \\
& \quad=\sum_{m=1}^{N} \frac{2}{J_{n+1}^{2}\left(j_{m}\right)}\left\{\left\langle f(t, \tau), \frac{1}{2 \pi} \int_{-R}^{R} e^{-s t} \tau J_{n}\left(j_{m} \tau\right) \Phi\left(s, j_{m}\right) d w\right\rangle\right\} .
\end{aligned}
$$

Since $f$ is a continuous linear functional, we have

$$
\begin{aligned}
& \sum_{m=1}^{N} \frac{2}{J_{n+1}^{2}\left(j_{m}\right)}\left\langle f(t, \tau), \frac{1}{2 \pi} \int_{-R}^{R} e^{-s t} \tau J_{n}\left(j_{m} \tau\right) \Phi\left(s, j_{m}\right) d w\right\rangle \\
& \quad=\left\langle f(t, \tau), \sum_{m=1}^{N} \frac{2}{J_{n+1}^{2}\left(j_{m}\right)} \frac{1}{2 \pi} \int_{-R}^{R} e^{-s t} \tau J_{n}\left(j_{m} \tau\right) \Phi\left(s, j_{m}\right) d w\right\rangle .
\end{aligned}
$$

Because $f \in L U_{a, b, c n}^{\prime}$ and in view of Lemma 5.5, the last expression tends to $\langle f(t, \tau\rangle, \tau \dot{\phi}(t, \tau)\rangle$ as $R, N \rightarrow \infty$. This completes our proof of the main theorem.

Uniqueness Theorem 6.1. If $\mathscr{L} \mathscr{H}_{n}(f)=F\left(s, j_{m}\right)$ and $\mathscr{L} \mathscr{H}_{n}(g)=$ $G\left(s, j_{m}\right)$ for all $s \in \Omega_{f}=\left\{s / \sigma_{f}<\operatorname{Re}(s)<\rho_{f}\right\}$ and $s \in \Omega_{g}=\{s / \sigma g<$ $\left.\operatorname{Re}(s)<\rho_{g}\right\}$ and $\left\{j_{m}\right\}$, positive zeros of $J_{n}(z)$, if $\Omega_{f} \cap \Omega_{g} \neq 0$, and if $F\left(s, j_{m}\right)=G\left(s, j_{m}\right)$ for $s \in \Omega_{f} \cap \Omega_{g}$, then $f=g$ in the sense of equality in $D^{\prime}(I)$.

Proof. By above theorem, in the sense of convergence in $D^{\prime}(I)$ 


$$
\begin{aligned}
f & =\lim _{R, N \rightarrow \infty}\left\{\frac{1}{2 \pi i} \sum_{m=1}^{N} \frac{2 J_{n}\left(j_{m} y\right)}{J_{n+1}^{2}\left(j_{m}\right)}\left[\int_{\sigma-i R}^{\sigma+i R} e^{s x} F\left(s, j_{m}\right) d s\right]\right\} \\
& ={ }_{R, N \rightarrow \infty}\left\{\frac{1}{2 \pi i} \sum_{m=1}^{N} \frac{2 J_{n}\left(j_{m} y\right)}{J_{n+1}^{2}\left(j_{m}\right)}\left[\int_{\sigma-i R}^{\sigma+i R} e^{s x} G\left(s, j_{m}\right) d s\right]\right\} \\
& =g(x, y) .
\end{aligned}
$$

Hence $f=g$, in the sense of equality in $D^{\prime}(I)$.

ACKnowledgment. The second author (R. A. P.) is grateful to Dr. M. S. Chaudhary for the help given by him during the preparation of this paper. The author also wishes to thank the referee for the valuable suggestions for the improvement of the paper.

\section{REFERENCES}

1. J. L. B. Cooper, Laplace transformation of distributions, Canad. J. Math., 18 (1966), 1323-1332.

2. L. S. Dube, On Finite Hankel transformation, Pacific J. Math., 62 (2) (1976), 365378.

3. L. S. Dube and J. N. Pandey, On the Hankel transform of distributions, Tôhoku Math. J., 27 (1975), 337-354.

4. J. N. Pandey, On the stieltjes transform of generalized functions, Proc. Cam. Phi. Soc., 75 (1972), 85-96.

5. J. N. Pandey and A. H. Zemanian, Complex inversion for the generalized convolution transform, Pacific J. Math., 5 (1968), 147-157.

6. G. N. Watson, Theory of Bessel Functions, Cambridge University Press, 1958.

7. A. H. Zemanian, Inversion formulas for the distributional Laplace transformation, SIAM J. Appl. Math., 14 (1966), 159-166.

8. — A generalized Weierstrass transformation, SIAM J. Appl. Math., 15 (1967), $1088-1105$.

9. —_, A distributional K-transformation, SIAM J. Appl. Math., 14 (1966), 13501365.

10. — Generalized Integral Transformations, Interscience Publishers, 1968.

Received February 23, 1978 and in revised form August 17, 1978.

MarathWADA UNIVERSITY

AURANGABAD, INDIA

AND

SHIVAJI UNIVERSITY

KOLHAPUR, INDIA 


\section{PACIFIC JOURNAL OF MATHEMATICS}

\section{EDITORS}

RICHARD ARENS (Managing Editor)

University of California

Los Angeles, CA 90024

Charles W. Curtis

University of Oregon

Eugene, OR 97403

C. C. MOORE

University of California

Berkeley, CA 94720
J. DUGUNDJI

Department of Mathematics

University of Southern California

Los Angeles, CA 90007

R. FINN and J. MILGRAM

Stanford University

Stanford, CA 94305

\section{ASSOCIATE EDITORS}

\section{E. F. BeCKENBACH}

B. H. NeumanN

F. WOLF

K. YoSHIDA

\section{SUPPORTING INSTITUTIONS}

\author{
UNIVERSITY OF SOUTHERN CALIFORNIA \\ STANFORD UNIVERSITY \\ UNIVERSITY OF HAWAII \\ UNIVERSITY OF TOKYO \\ UNIVERSITY OF UTAH \\ WASHINGTON STATE UNIVERSITY \\ UNIVERSITY OF WASHINGTON
}

The Supporting Institutions listed above contribute to the cost of publication of this Journal, but they are not owners or publishers and have no responsibility for its content or policies.

Mathematical papers intended for publication in the Pacific Journal of Mathematics should be in typed form or offset-reproduced, (not dittoed), double spaced with large margins. Please do not use built up fractions in the text of the manuscript. However, you may use them in the displayed equations. Underline Greek letters in red, German in green, and script in blue. The first paragraph or two must be capable of being used separately as a synopsis of the entire paper. Items of the bibliography should not be cited there unless absolutely necessary, in which case they must be identified by author and journal, rather than by item number. Manuscripts, in triplicate, may be sent to any one of the editors. Please classify according to the scheme of Math. Reviews, Index to Vol. 39. All other communications should be addressed to the managing editor, or Elaine Barth, University of California, Los Angeles, California, 90024.

50 reprints to each author are provided free for each article, only if page charges have been substantially paid. Additional copies may be obtained at cost in multiples of 50 .

The Pacific Journal of Mathematics is issued monthly as of January 1966. Regular subscription rate: $\$ 72.00$ a year (6 Vols., 12 issues). Special rate: $\$ 36.00$ a year to individual members of supporting institutions.

Subscriptions, orders for numbers issued in the last three calendar years, and changes of address should be sent to Pacific Journal of Mathematics, P.O. Box 969, Carmel Valley, CA 93924, U.S.A. Older back numbers obtainable from Kraus Periodicals Co., Route 100, Millwood, NY 10546.

PUBLISHED BY PACIFIC JOURNAL OF MATHEMATICS, A NON-PROFIT CORPORATION

Printed at Kokusai Bunken Insatsusha (International Academic Printing Co., Ltd.). 8-8, 3-chome, Takadanobaba, Shinjuku-ku, Tokyo 160, Japan. 


\section{Pacific Journal of Mathematics}

\section{Vol. 80, No. $2 \quad$ October, 1979}

K. Adachi, On the multiplicative Cousin problems for $N^{p}(D) \ldots \ldots \ldots \ldots 297$

Howard Banilower, Isomorphisms and simultaneous extensions in $C(S) \ldots 305$

B. R. Bhonsle and R. A. Prabhu, An inversion formula for a distributional

finite-Hankel-Laplace transformation ................... 313

Douglas S. Bridges, Connectivity properties of metric spaces.......... 325

John Patton Burgess, A selection theorem for group actions ........... 333

Carl Claudius Cowen, Commutants and the operator equations

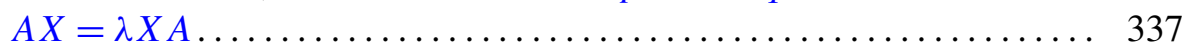

Thomas Curtis Craven, Characterizing reduced Witt rings. II .......... 341

J. Csima, Embedding partial idempotent d-ary quasigroups ............ 351

Sheldon Davis, A cushioning-type weak covering property ............ 359

Micheal Neal Dyer, Nonminimal roots in homotopy trees ............. 371

John Erik Fornaess, Plurisubharmonic defining functions ........... 381

John Fuelberth and James J. Kuzmanovich, On the structure of finitely

generated splitting rings .......................... 389

Irving Leonard Glicksberg, Boundary continuity of some holomorphic

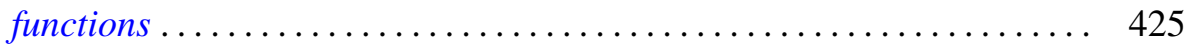

Frank Harary and Robert William Robinson, Generalized Ramsey theory.

IX. Isomorphic factorizations. IV. Isomorphic Ramsey numbers .......

Frank Harary and Allen John Carl Schwenk, The spectral approach to determining the number of walks in a graph...........

David Kent Harrison, Double coset and orbit spaces ..... . .

Shiro Ishikawa, Common fixed points and iteration of commuting

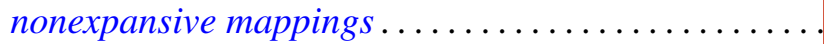

Philip G. Laird, On characterizations of exponential polynomials ........ 503

Y. C. Lee, A Witt's theorem for unimodular lattices ...........

Teck Cheong Lim, On common fixed point sets of commutative

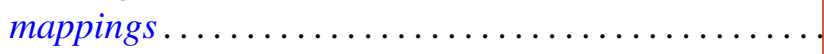

R. S. Pathak, On the Meijer transform of generalized functions ...

T. S. Ravisankar and U. S. Shukla, Structure of $\Gamma$-rings . . .

Olaf von Grudzinski, Examples of solvable and nonsolvable convolution

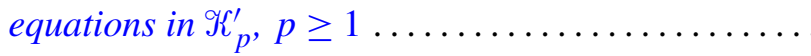

